\title{
International Journal of Sports and Exercise Medicine
}

Case Report: Open Access

\section{Paediatric Hip Pain: A Rare Injury}

\section{Raj Subbu* and Fares Haddad}

\author{
Institute of Sport Exercise and Health, UK
}

*Corresponding author: Raj Subbu, Clinical Research Fellow, Institute of Sport Exercise and Health, 170 Tottenham
Court Rd, London, W1T 7HA, UK, Tel: 07841575 312, E-mail: rajsubbu1@hotmail.com

\begin{abstract}
Paediatric hip pain in young athletes can be a diagnostic challenge for clinicians. The anatomy of the paediatric pelvis and surrounding structures varies considerably to that of an adult and therefore is predisposed to different types of injuries which can lead to future deformities if not managed appropriately. Unlike adult pelvic fractures, which have set guidelines and management strategies, less research exists on these injuries in the paediatric context and more specifically in the sporting environment. We present two cases with very different mechanisms of action sustaining a fracture through the triradiate cartilage. Both injuries were diagnosed early using plain radiographs and magnetic resonance imaging and treated non-operatively with regular follow-up and repeat radiographic investigations to assess fracture healing in order to structure the rehabilitation program and weight bearing status. At first follow-up (average 7 weeks) evidence of callus formation was seen with symptoms of pain much improved following conservative, non-weight bearing management. Despite the mechanism of injury, the clinician should have a high index of suspicion for this type of fracture, if the diagnosis is delayed this may lead to gross anatomical deformities requiring surgical intervention for correction, potentially preventing further physical activity and sports participation in the future.
\end{abstract}

\section{Introduction}

In the young athlete, injuries to the hip and pelvis are increasingly being recognised [1]. Hip injuries include both osseous and soft tissue aetiologies as a result of a single traumatic event, repetitive micro-trauma or underlying paediatric hip disorders such as slipped capital femoral epiphysis and Legge-Perthes disease [1]. At present, the literature surrounding these injuries has focussed on the common soft tissue injuries and apophyseal avulsion fractures $[1,2]$. With advances in diagnostic and therapeutic interventions, less common injuries are more frequently being recognised and managed. Acetabular fractures in children and adolescents are rare injuries, but ones that should not be missed as this may result in debilitating consequences.

The anatomy of the paediatric pelvis differs considerably to that of an adult; given the greater malleable structure and joint elasticity, more displacement can occur allowing for single fractures within the pelvic ring [3]. Furthermore, is the presence of the triradiate cartilage, which if damaged in children can lead to future skeletal abnormalities and growth disturbances [4]. Reported cases and outcomes of triradiate cartilage injuries remain limited in the literature. We present two cases of triradiate fractures with very different presentations and mechanisms of injury.

\section{Case One}

A 16-year-old male, first presented after 3 months of on-going pain in the left hip following a heavy fall diving playing soccer, landing onto his left buttock. His pain progressed to left pelvic and groin pain. Initially he was able to continue to training but after thirty minutes following another fall from diving he developed anterior groin pain. He had initially been managed with physiotherapy with off-loading, re-conditioning and time off sport to allow the pain to settle. However whilst attempting to return to play he was unable to complete training with persistent anterior groin pain. There was no pain in the lumbar spine or knees. On clinical examination he walked with an antalgic gait. Range of movement in the left hip was restricted due to pain. There was pain on palpation over the left anterior inferior iliac spine with weakness of resisted hip flexion. There were no cross over signs and the neurovascular assessment was normal.

The initial working diagnosis was a rectus femoris type avulsion fracture that had not healed. Initial radiographs were unremarkable shown in figure 1. An MRI was requested, shown in figure 2 indicating extensive bone marrow oedema involving the left superior

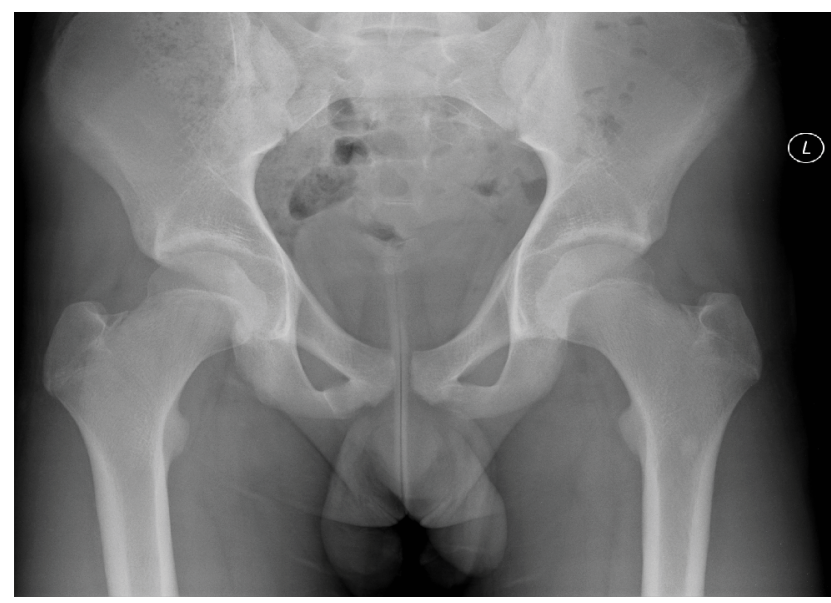

Figure 1: The initial plain radiograph confirms asymmetry of the left superio pubic ramus. There is a peri-osteal reaction and there is a soft tissue mass seen at the medial aspect of the pubic ramus.

\section{Clinmed International Library}

Citation: Subbu R, Haddad F (2015) Paediatric Hip Pain: A Rare Injury. Int J Sports Exerc Med 1:015

Received: June 27, 2015: Accepted: August 03, 2015: Published: August 07, 2015 Copyright: @ 2015 Subbu R. This is an open-access article distributed under the terms of the Creative Commons Attribution License, which permits unrestricted use, distribution, and reproduction in any medium, provided the original author and source are credited. 

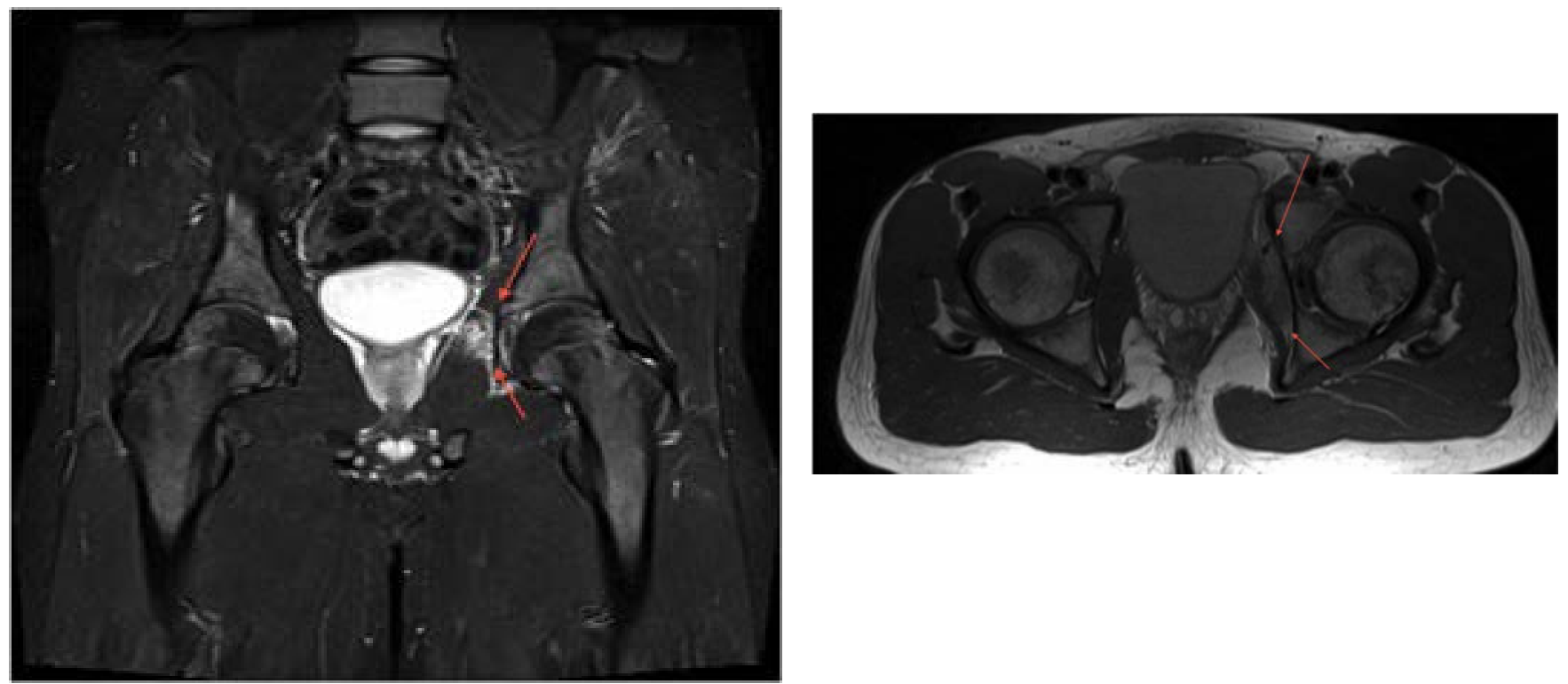

Figure 2: Initial MRI investigations. Extensive bone marrow oedema involving the left superior pubic ramus extending proximally to border the triradiate cartilage is illustrated
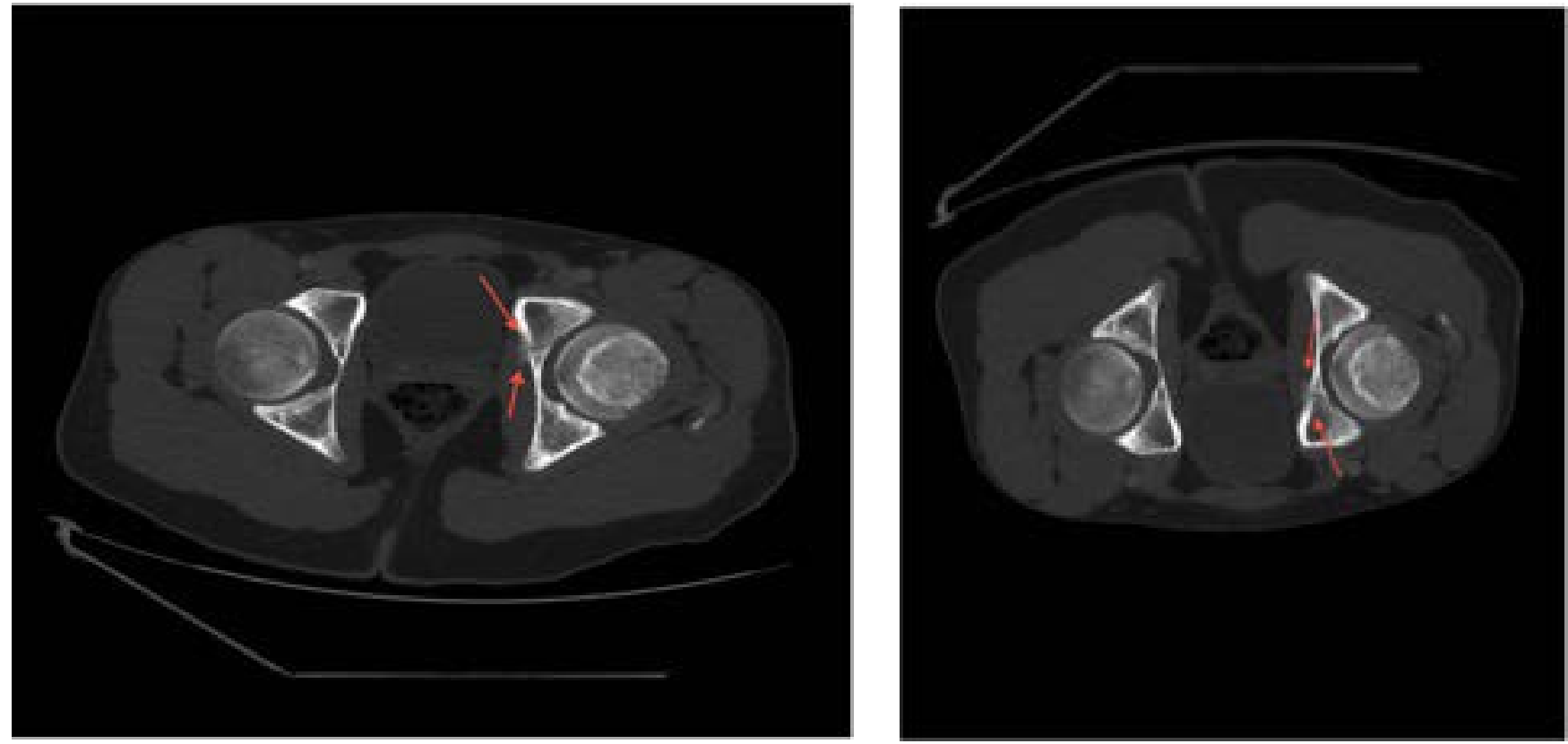

Figure 3: The CT scan confirms an abnormality of the left triradiate cartilage, which shows a fracture. This is breaching the medial wall of the acetabulum and is extending cranially to breach the superior margin of the superior pubic ramus. There is an organised peri-osteal reaction. There is a band of marginal callus around the medial wall of the medial fracture there is further organising callus around the anterior margin of the fracture.

pubic ramus extending proximally to border the triradiate cartilage. There is high signal within the triradiate cartilage. There is a periosteal reaction of the anterior margin of the superior pubic ramus. There is a soft tissue mass extending posteriorly along the medial wall of the acetabulum. This is causing displacement of the obturator internus with an associated effusion in the hip.

Given the findings a CT, figure 3, was requested to rule underlying pathological cause. The images are consistent with recurrent trauma to triradiate cartilage and pubis. The management was to further offload the hip joint completely from any jumping or diving to that side with a repeat MRI at 6 weeks to further rule out a pathological cause. At six weeks a repeat MRI performed to assess the healing process, shown in figure 4. At this stage the patient was pain free with good range of movement in hip examination and a gradual weight bearing and rehabilitation program developed with full training resumed at a further 3 months.

\section{Case Two}

An 11-year-old girl presented to the Emergency Department complaining of pain in her right hip. She had sustained a mechanical, low impact fall during a school field trip falling onto concrete flooring, landing directly onto the lateral aspect of the right hip, she was unable to weight bear immediately after. There were no symptoms of pain prior to the fall and her previous medical history was unremarkable.

Clinical examination of the hip was limited due to pain and all movements were restricted. Lumbar spine, knee examinations and neurovascular assessment were normal. Vital observations were normal and she was apyrexial. Routine blood results were normal. Initial plain radiographs indicated an un-displaced fracture through the acetabulum, shown in figure 5.

The initial management plan was non-weight bearing with regular follow-up and imaging to assess healing. At first follow-up 

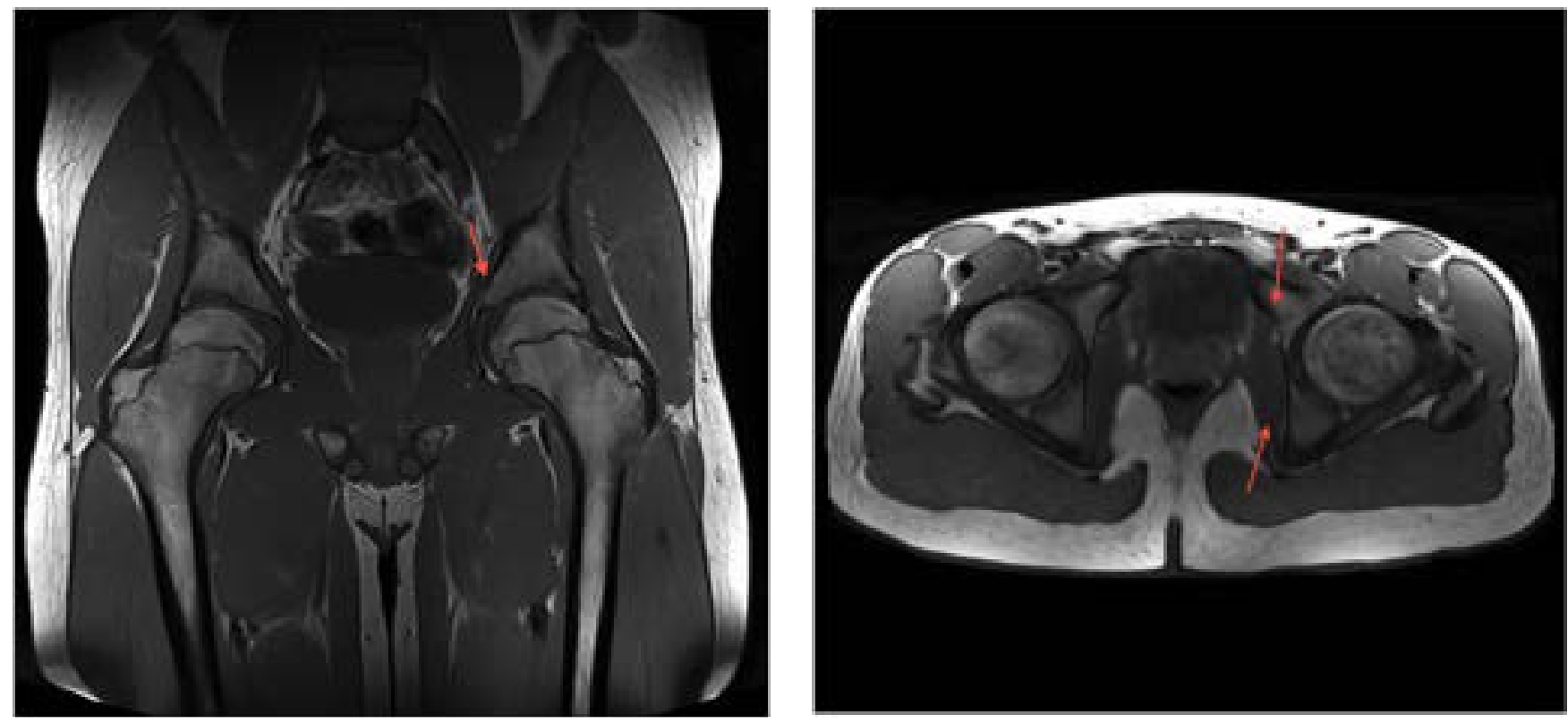

Figure 4: The fracture through the triradiate cartilage shows much improvement. The extra-osseous mass has resolved and there is some callus formation and peri-osteal reaction around the medial margin of the fracture deep to the obturator internus.
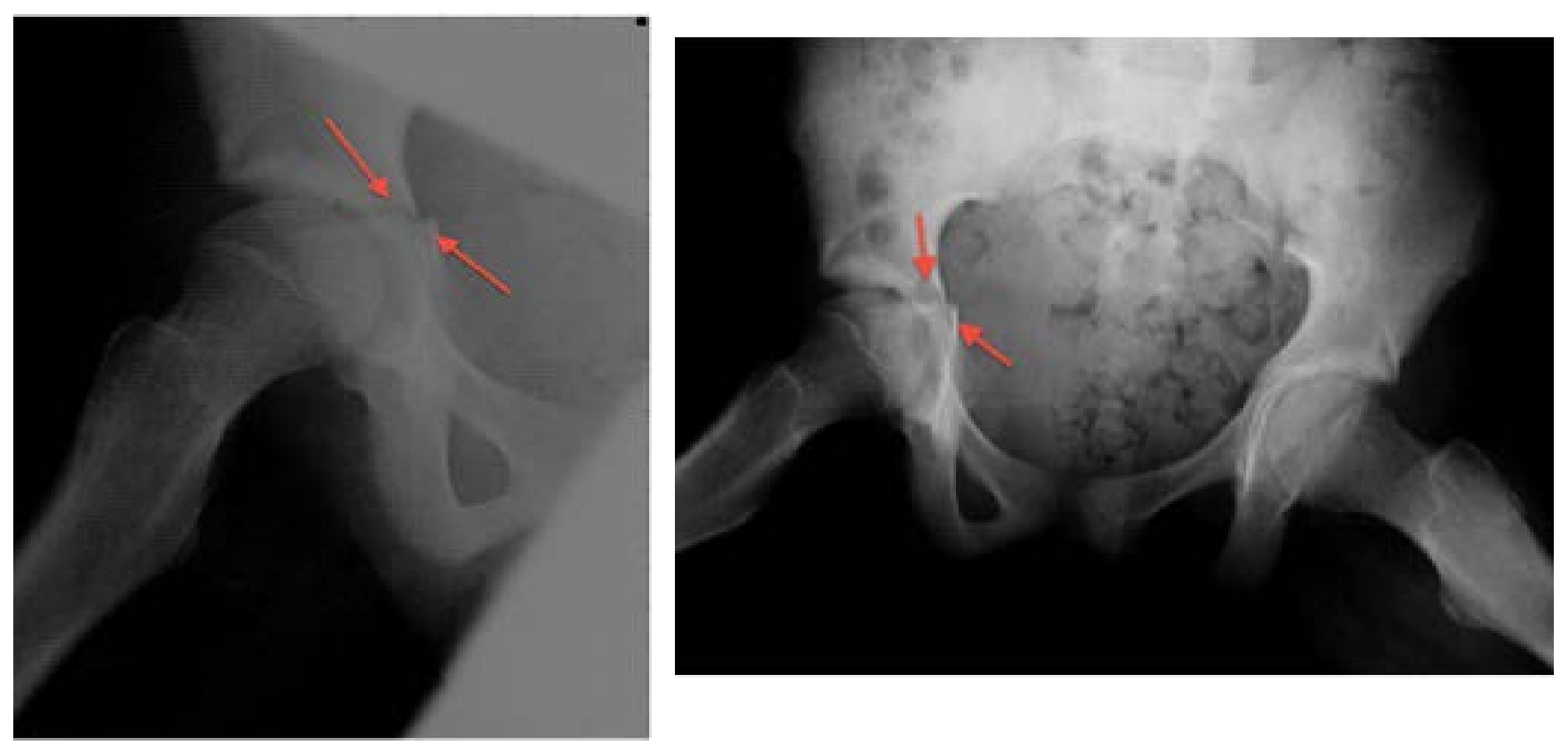

Figure 5: Indicating un-displaced fracture through acetabulum with discontinuity of the medial aspect of the right acetabular contour.

in 2 weeks there was persistent pain an MRI was requested to assess the injury further shown in figure 6; which illustrated the fracture through the triradiate cartilage.

As the fracture was minimally displaced conservative management was followed with further clinical improvement seen at 6 weeks. Toetouch weight bearing was achieved by 3 months progressing to full weight bearing status by 5 months. At 6 months repeat radiographs were taken, shown in figure 7 showing good alignment and callus formation. The patient continued to improve, was pain free and range of movements improving on examination.

\section{Discussion}

Paediatric hip trauma and associated pain can pose a diagnostic challenge and an understanding of the developing paediatric pelvic anatomy is required to broaden the physicians differential diagnosis. The causes of hip pathology are often related to skeletal immaturity and are fairly specific to the age of the child. The initial presentation may include a temporary decline in coordination and musculoskeletal balance as limb length increases at a faster rate during puberty than does limb mass [5]. As a result of this the hip flexors, extensors and hamstrings may become inflexible predisposing the paediatric athlete to muscle injury and avulsion fractures ${ }^{1}$. Furthermore, the open physes and growing cartilage are more susceptible to injury and shear forces that can result in premature physeal arrest, apophyseal avulsion fractures, and chondral injuries [6].

The anatomy and the development of the acetabulum is complex and poorly understood [7]. The triradiate cartilage is formed by an ilio-ischial flange posteriorly, an iliopubic flange anteriorly, and an ischio- pubic flange inferiorly. Its borders are the three sites of primary growth of the acetabulum and its growth and re-modeling ensure that the diameter of the acetabulum enlarges proportionally with the growing femoral head [8]. Growth plates on all sides surround the three bones, which form the acetabulum, medially. These face towards the centre of the triradiate cartilage, the limbs of the triradiate cartilage and the articular surface. 

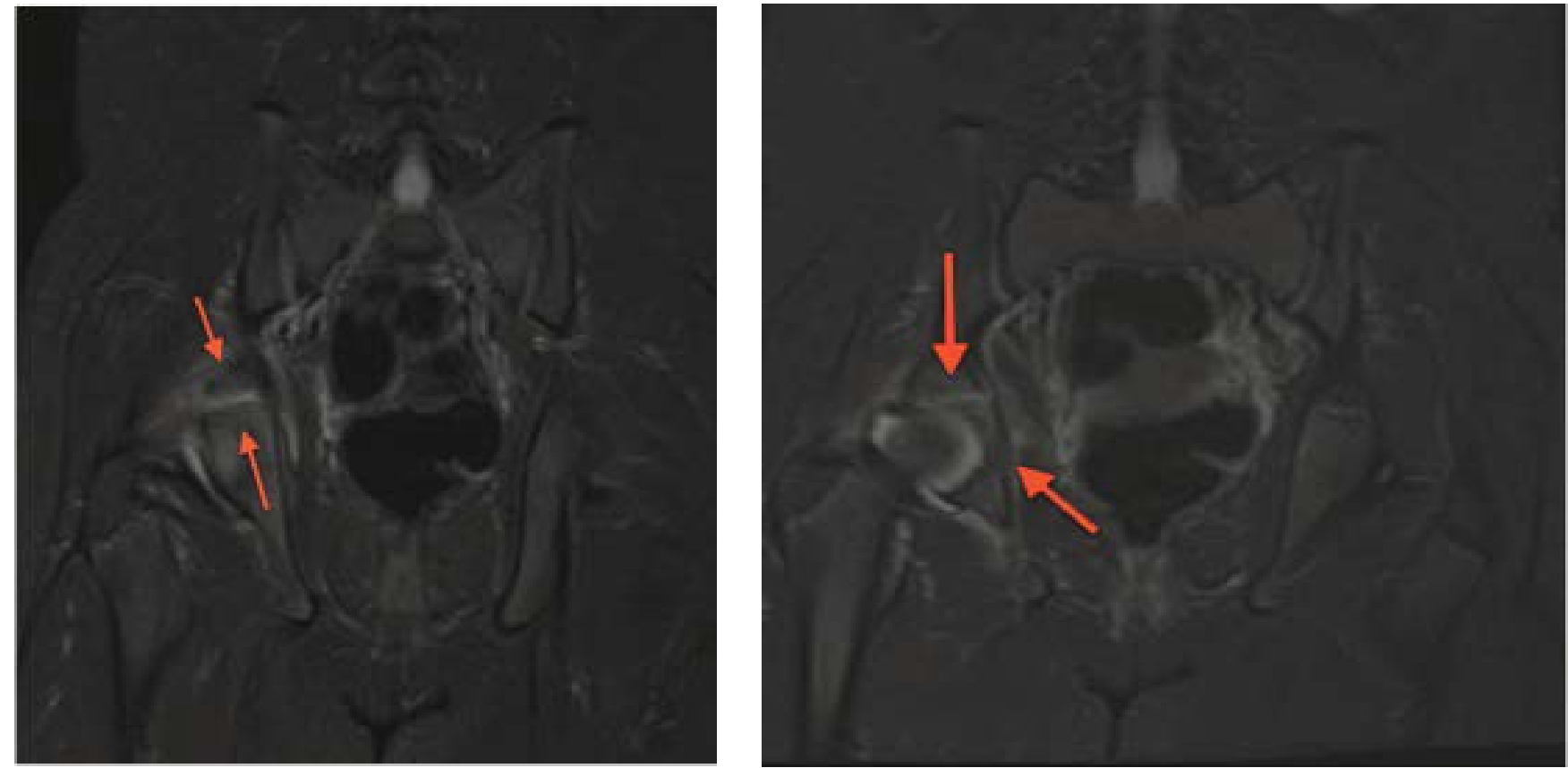

Figure 6: There is an undisplaced fracture through the triradiate cartilage of the right hip when compared to the left. This has breached the articular margin of the joint. There is bone marrow oedema of each of the bony margins bordering the triradiate cartilage.

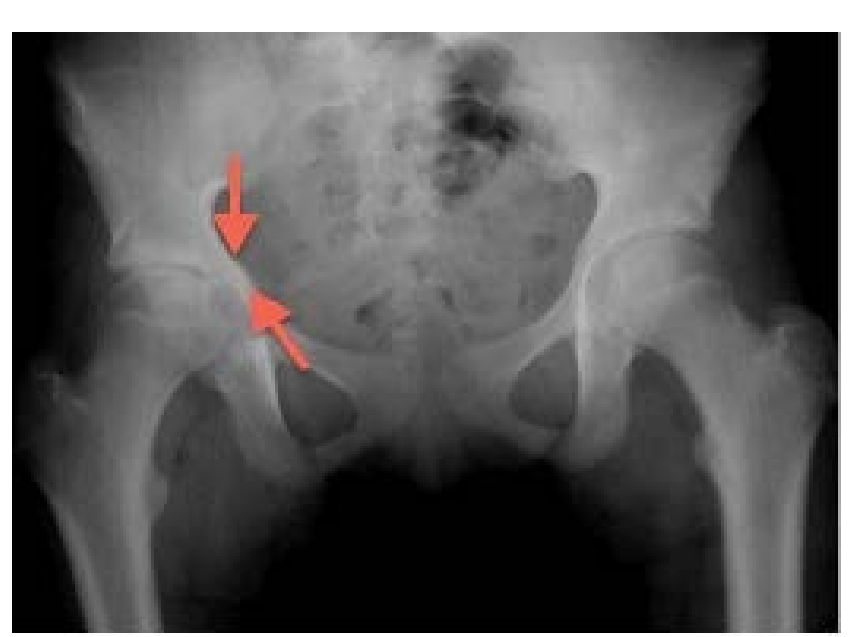

Figure 7: Repeat radiographs at 8 weeks show good alignment and signs of callus formation.

Each may be divided into four distinct areas according to the orientation of its cell columns, which reflect the direction of growth [9].

Injuries to the triradiate cartilage may result in a number of morphologic and clinical complications. If the acetabular physis remains intact but the triradiate closes, disproportionate growth can result in lateralization of the hip joint secondary to a thickened medial wall $[10,11]$. This shallow acetabulum with resultant high acetabular index can manifest by lateral subluxation/dislocations of the femoral head [11] resulting in osteonecrosis [12]. As development continues, anteversion of the femoral neck increases to avoid recurrent dislocations [12]. The clinical manifestation of interrupted growth and the resultant acetabular dysplasia are pain, gait disturbance, and joint degeneration with progression to early onset of degenerative joint disease [12]. It remains difficult to predict which patients with an injured triradiate cartilage will subsequently develop traumatic acetabular dysplasia, partial fusion of the physis, and further growth disturbances with clinical symptoms $[13,14]$. The growth potential of the physis decreases with age [12], the younger the patient at the time of premature fusion of the triradiate cartilage, the greater the expected deformity [15]. Bucholz et al. [16] proposed a classification scheme based on the Salter-Harris classification whereby triradiate injuries would parallel Salter-Harris injuries I, II and V [16]. According to this scheme, a type I or II injury does not disrupt the germinal zone, and therefore the blood supply remains intact and growth continues, whereas a type $\mathrm{V}$ crushing injury might damage the bipolar zones of the physis, affecting the blood supply and growth. More reference

Acetabulum fractures in children are rare and can occur as isolated injuries or associated with pelvic or long bone fractures [17]. The mechanism tends to be related to high-energy trauma, for example in a road traffic accident. Due to the skeletal immaturity and flexibility in the paediatric setting reports of this type of injury following minor trauma is limited [18]. The exact mechanism of an acetabular fracture in a child due to minor/low-energy trauma remains unclear and poorly understood. The external forces causing pelvic disruption in children were studied and classified by McDonald as lateral compression, anteroposterior compression, and vertical shear. With lateral compression, the triradiate cartilage in the child is at greater risk for disruption and displacement [19].

The treatment options for acetabular fractures are divided into operative and non-operative. In adults, treatment indications are clear with extensive evidence based management on acetabula fractures. However, limited research exists on acetabula fractures in children, particularly in the paediatric athlete $[8,20]$.

The current literature supports non-operative management with surgical management reserved for severely displaced fractures and instability [21]. Two previous cases are reported of adolescent males suffering this injury following low energy trauma whilst playing sport $[22,23]$. Both injuries were diagnosed using CT and MRI images and treated non-operatively with signs of fracture healing at 8 weeks and return to sport within 12 months.

The two cases presented here illustrate the diagnostic challenge of these injuries. We highlight the variable nature of the mechanism and low energy type of injury and emphasise the need for a high index of suspicion by the clinician with hip pain, especially in those which are not resolving and where plain radiographs appear unremarkable. The management is consistent with current literature and we further stress the importance of further radiological imaging including CT and/or MRI for exact diagnosis, fracture pattern, stability and displacement. Closely monitored follow-up in conjunction with radiological imaging should be utilized to determine fracture healing combined with clinical assessment to increase weight-bearing status and allow for full recovery. 


\section{Conclusion}

The major problem with this type injury is the limited evidence available to gold standard treatment. As a result the treatment plans vary with no consistent guidelines or algorithms. When disruption of the triradiate cartilage occurs in young children, it may lead to premature physeal closure and later deformities.

The clinician needs to be aware of the immature skeletal anatomy and take into consideration the patient's age when interpreting the clinical and radiological findings. In these cases the mechanism, although very different, resulted in the same injury. With regards to hip pain in the paediatric athlete one should be aware of this fracture pattern regardless of the low level energy of trauma. With greater access to imaging modalities available, early detection and diagnosis is key to providing the appropriate management plan to prevent future complications.

\section{References}

1. Frank JS, Gambacorta PL, Eisner EA (2013) Hip pathology in the adolescent athlete. J Am Acad Orthop Surg 21: 665-674.

2. Kocher MS, Tucker R (2006) Pediatric athlete hip disorders. Clin Sports Med 25: $241-253$.

3. Watts HG (1976) Fractures of the pelvis in children. Orthop Clin North Am 7: 615-624.

4. Heeg M, Visser JD, Oostvogel HJ (1988) Injuries of the acetabular triradiate cartilage and sacroiliac joint. J Bone Joint Surg $\mathrm{Br} 70$ : 34-37.

5. Hawkins D, Metheny J (2001) Overuse injuries in youth sports: biomechanical considerations. Med Sci Sports Exerc 33: 1701-1707.

6. Kovacevic D, Mariscalco M, Goodwin RC (2011) Injuries about the hip in the adolescent athlete. Sports Med Arthrosc 19: 64-74.

7. Portinaro NM, Murray DW, Benson MK (2001) Microanatomy of the acetabular cavity and its relation to growth. J Bone Joint Surg $\mathrm{Br} 83$ : 377-383.

8. Heeg M, de Ridder VA, Tornetta P 3rd, de Lange S, Klasen HJ (2000) Acetabular fractures in children and adolescents. Clin Orthop Relat Res: 80-86.

9. Badgley C (1943) Correlation of clinical and anatomical facts leading to a conception of the aetiology of congenital hip dysplasias. J Bone Joint Surg 24: $503-523$
10. Trousdale RT, Ganz R (1994) Posttraumatic acetabular dysplasia. Clin Orthop Relat Res: 124-132.

11. Hallel T, Salvati EA (1977) Premature closure of the triradiate cartilage. A case report and animal experiment. Clin Orthop Relat Res: 278-281.

12. Ponseti IV (1978) Growth and development of the acetabulum in the norma child. Anatomical, histological, and roentgenographic studies. J Bone Joint Surg Am 60: 575-585.

13. Rodrigues KF (1973) Injury of the acetabular epiphysis. Injury 4: 258-260.

14. Blair W, Hanson C (1979) Traumatic closure of the triradiate cartilage: report of a case. J Bone Joint Surg Am 61: 144-145.

15. Scuderi G, Bronson MJ (1987) Triradiate cartilage injury. Report of two cases and review of the literature. Clin Orthop Relat Res: 179-189.

16. Bucholz RW, Ezaki M, Ogden JA (1982) Injury to the acetabular triradiate physeal cartilage. J Bone Joint Surg Am 64: 600-609.

17. Weisel A, Hecht HL (1980) Occult fracture through the triradiate cartilage of the acetabulum. AJR Am J Roentgenol 134: 1262-1264.

18. Currey JD, Butler G (1975) The mechanical properties of bone tissue in children. J Bone Joint Surg Am 57: 810-814.

19. McDonald GA (1980) Pelvic disruptions in children. Clin Orthop Relat Res: 130-134.

20. Heeg M, de Ridder VA, Tornetta P $3^{\text {rd }}$, de Lange S, Klasen HJ (2000) Acetabular fractures in children and adolescents. Clin Orthop Relat Res: 80-86.

21. Valdiserri L, Bungaro P, D'Angelo G (1996) Traumatic lesions of the acetabular triradiate cartilage (presentation of four cases and considerations on treatment). Chir Organi Mov 81: 361-367.

22. McDonnell M, Schachter AK, Phillips DP, Liporace FA (2007) Acetabular fracture through the triradiate cartilage after low-energy trauma. J Orthop Trauma 21: 495-498.

23. Ergen FB, Yildiz AE, Ayvaz M, Aydingoz U (2012) Bilateral triradiate cartilage injury: an overuse syndrome in an adolescent football player. Skeletal Radio 41: 353-355 